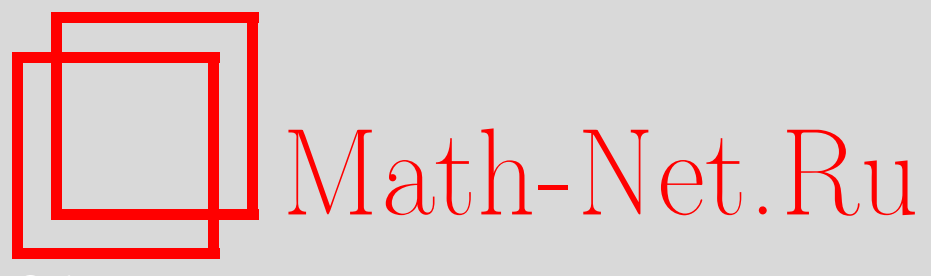

А. С. Демидов, Д. А. Платущихин, Явная формула для градиента гармонической функции по ее аналитическим данным Коши на аналитической кривой, Матем. заметки, 2010, том 87, выпуск 1, 141-143

DOI: https://doi.org/10.4213/mzm8553

Использование Общероссийского математического портала Math-Net.Ru подразумевает, что вы прочитали и согласны с пользовательским соглашением http: //www . mathnet.ru/rus/agreement

Параметры загрузки:

IP: 54.172 .240 .79

26 апреля 2023 г., 16:18:36

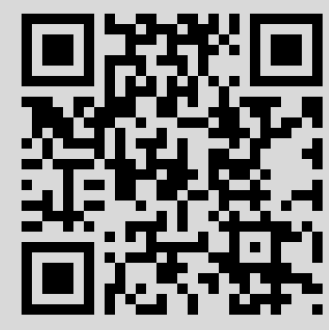




\section{Явная формула для градиента гармонической функции по ее аналитическим данным Коши на аналитической кривой}

\section{А. С. Демидов, Д. А. Платущихин}

Различные аспекты задачи Коши для двумерного уравнения Лапласа

$$
\Delta u=0 \quad \text { в } V_{\Gamma},\left.\quad u\right|_{P_{s} \in \Gamma}=F(s),\left.\quad \frac{\partial u}{\partial \nu}\right|_{P_{s} \in \Gamma}=G(s),
$$

в некоторой окрестности $V_{\Gamma}$ аналитической кривой $\Gamma$ изучались в работах многих авторов (см., например, [1]-[3]). В данной заметке мы предполагаем, что аналитическая кривая $\Gamma$ длины $2 \pi$ является границей односвязной области $\Omega \subset \mathbb{R}^{2} \simeq \mathbb{C}, \nu$-это внешняя (относительно области $\Omega$ ) единичная нормаль в точке $P_{s} \in \Gamma$, а

$$
F: s \mapsto F(s)=\operatorname{Re} \sum_{k \geqslant 0} f_{k} e^{i k s} \quad \text { и } \quad G: s \mapsto G(s)=\operatorname{Re} \sum_{k \geqslant 0} g_{k} e^{i k s}
$$

- это вещественнозначные аналитические функции натурального параметра $s \in \mathbb{R} / 2 \pi$, соответствующего точке $P_{s} \in \Gamma$. Кривая Г полностью определяется заданием $2 \pi$-периодической функции

$$
Q: \mathbb{T}=\mathbb{R} / 2 \pi \ni s \mapsto Q(s)=N(s)-s,
$$

где $N(s)$ - угол между осью абсцисс и нормалью $\nu$ к $\Gamma$ в точке $P_{s} \in \Gamma$. Как известно, (см., например, [4; с. 137]), коэффициенты $L_{k}$ и $M_{k}$ в Фурье-представлении

$$
Q(s)=L_{0}+\sum_{k \geqslant 1}\left(L_{k} \cos k s+M_{k} \sin k s\right)
$$

аналитической функции $Q$ допускают оценки

$$
\left|L_{k}\right| \leqslant C e^{-k \tau}, \quad\left|M_{k}\right| \leqslant C e^{-k \tau},
$$

где константа $C$ мажорирует в $\tau$-окрестности кривой $\Gamma$ модуль аналитической функции $\mathscr{Q}$, ограничением которой на Г является функция $Q$.

Ниже выводятся формулы, задающие решение $u$ задачи (1) и его градиент в виде степенных рядов с коэффициентами, явно зависящими от параметров $f_{k}, g_{k}, L_{k}$ и $M_{k}$, фигурирующих в формулах (2), (3). Это позволяет вычислить с любой степенью точности как решение, так и градиент решения этой некорректной (по Адамару) задачи. Выведенные здесь формулы дают также возможность свести обратную задачу со свободной границей для уравнения Грэда-Шафранова (в цилиндрическом приближении) к соответствующей задаче в фиксированной области (см., например, [5]).

Отметим, прежде всего, что формула

$$
V_{\mathbb{T}} \ni \rho e^{i \theta} \mapsto U(\rho, \theta) \stackrel{(2)}{=} \operatorname{Re}\left(f_{0}+g_{0} \ln \rho+\frac{1}{2} \sum_{k \geqslant 1}\left\{\left(f_{k}+\frac{g_{k}}{k}\right) \rho^{k}+\left(f_{k}-\frac{g_{k}}{k}\right) \rho^{-k}\right\} e^{i k \theta}\right)
$$

задает в некоторой (двухсторонней) окрестности $V_{\mathbb{T}} \subset \mathbb{C}$ единичной окружности $\mathbb{T}$ решение такой задачи:

$$
\Delta U=0 \quad \text { в } \quad V_{\mathbb{T}},\left.\quad U(\rho, \theta)\right|_{\rho=1}=F(\theta),\left.\quad \frac{\partial U(\rho, \theta)}{\partial \rho}\right|_{\rho=1}=G(\theta) .
$$

Работа выполнена при поддержке Российского фонда фундаментальных исследований (грант № 07-01-00500).

(C) А. С. ДЕмидов, Д. А. ПлАтущихин, 2010 
В нижеследующей теореме 1 доказывается существование однолистного отображения $\zeta: V_{\Gamma} \ni z \mapsto \zeta(z) \in V_{\mathbb{T}}$ некоторых окрестностей кривой $\Gamma$ и окружности $\mathbb{T}$, для которого $|d \zeta / d z| \equiv 1$ на $\Gamma$, иначе говоря, отображение $\zeta$ изометрично на $\Gamma$. Тем самым, формула

$$
u(x, y)=U(\rho(x, y), \theta(x, y)), \quad \text { где } \quad \rho(x, y) e^{i \theta(x, y)}=\zeta(x+i y),
$$

задает решение задачи (1).

Tеорема 1 (ср. [6], [7]). В некоторой (двухсторонней) окрестности $V_{\mathbb{T}} \subset \mathbb{C}$ единичной окружности $\mathbb{T}$ определено однолистное отображение

$$
z: V_{\mathbb{T}} \ni \zeta=\rho e^{i \theta} \mapsto z(\zeta)=P_{0}+\int_{1}^{\zeta} \exp (A+i B) d \zeta \in V_{\Gamma}=z\left(V_{\mathbb{T}}\right), \quad P_{0} \in \Gamma .
$$

Здесь $A+i B=\ln d z / d \zeta-$ аналитическая функция комплексного переменного $\zeta$, задаваемая решением следующей задачи Коши:

$$
\left.B(\rho, \theta)\right|_{\rho=1}=N(\theta)-\theta,\left.\quad A\right|_{\rho=1}=0 \quad\left(\left.\Rightarrow \frac{\partial B}{\partial \rho}\right|_{\rho=1}=0\right), \quad \rho=|\zeta|, \quad \theta=\arg \zeta .
$$

А именно,

$$
\begin{aligned}
& A: V_{\mathbb{T}} \ni \zeta=\rho e^{i \theta} \mapsto A(\rho, \theta)=\frac{1}{2} \sum_{k \geqslant 1}\left(\rho^{k}-\rho^{-k}\right)\left\{M_{k} \cos k \theta-L_{k} \sin k \theta\right\}, \\
& B: V_{\mathbb{T}} \ni \zeta=\rho e^{i \theta} \mapsto B(\rho, \theta)=L_{0}+\frac{1}{2} \sum_{k \geqslant 1}\left(\rho^{k}+\rho^{-k}\right)\left\{L_{k} \cos k \theta+M_{k} \sin k \theta\right\},
\end{aligned}
$$

где $L_{k}$ и $M_{k}$ - коэффициенты, фигурирующие в формуле (3). При этом отображение (5) изометрично на $\mathbb{T}$, m.e.

$$
\left|\frac{d z(\zeta)}{d \zeta}\right| \equiv 1 \quad \partial \Omega \Omega \quad \zeta \in \mathbb{T} .
$$

Доказательство. В силу оценок (4) формулы (7), (8) задают в некоторой окрестности окружности $\mathbb{T}$ аналитическую функцию $A+i B$, а тем самым, и отображение (5). Имеем: $B \stackrel{(5)}{=} \arg d z / d \zeta$, т.е. $\left.B(\rho, \theta)\right|_{\rho=1}=N(s(\theta))-\theta$, где $s(\theta)$ - длина дуги кривой $\Gamma$, параметризованная точкой $e^{i \theta} \in \mathbb{T}$. Изометрия между $\mathbb{T}$ и $\Gamma$ эквивалентна условию $\left.e^{A}\right|_{\rho=1} \equiv 1$, ибо $\left.e^{A}\right|_{\rho=1}=d s(\theta) / d \theta$. Без ограничения общности можно считать, что $s(\theta)=\theta$. Тем самым, $N(s(\theta))=N(\theta)$ равносильно $\left.B(\rho, \theta)\right|_{\rho=1}=N(\theta)-\theta$.

Теорема 2. Справедливы следующие формуль:

$$
\begin{aligned}
& \frac{\partial u}{\partial x}(x(\rho, \theta), y(\rho, \theta))=\left(\frac{\partial U(\rho, \theta)}{\partial \rho} \cos N(\rho, \theta)-\frac{1}{\rho} \frac{\partial U(\rho, \theta)}{\partial \theta} \sin N(\rho, \theta)\right) e^{-A(\rho, \theta)} \\
& \frac{\partial u}{\partial y}(x(\rho, \theta), y(\rho, \theta))=\left(\frac{\partial U(\rho, \theta)}{\partial \rho} \sin N(\rho, \theta)+\frac{1}{\rho} \frac{\partial U(\rho, \theta)}{\partial \theta} \cos N(\rho, \theta)\right) e^{-A(\rho, \theta)}
\end{aligned}
$$

Здесъ $x(\rho, \theta)+i y(\rho, \theta) \stackrel{(5)}{=} z\left(\rho e^{i \theta}\right), A(\rho, \theta)$ и B( $\left.\rho, \theta\right)$ представлены формулами (7) u (8), а

$$
\begin{aligned}
& \frac{\partial U}{\partial \rho}=\frac{1}{2} \operatorname{Re} \sum_{k \geqslant 0}\left\{k f_{k}\left(\rho^{k-1}-\rho^{-k-1}\right)+g_{k}\left(\rho^{k-1}+\rho^{-k-1}\right)\right\} e^{i k \theta}, \\
& \frac{\partial U}{\partial \theta}=\frac{1}{2} \operatorname{Im} \sum_{k \geqslant 1}\left\{k f_{k}\left(\rho^{k}+\rho^{-k}\right)+g_{k}\left(\rho^{k}-\rho^{-k}\right)\right\} e^{i k \theta}
\end{aligned}
$$

где $f_{k}$ и $g_{k}-$ коэффициенты, фигурирующие в формулах (2). 
ДоказАтеЛЬСтво. Имеем

$$
\zeta=\xi+i \eta, \quad \xi=\rho \cos \theta, \quad \eta=\rho \sin \theta, \quad \frac{d z}{d \zeta} \stackrel{(5)}{=} e^{A+i B} .
$$

Поэтому

$$
\frac{\partial x}{\partial \xi}=e^{A} \cos B, \quad \frac{\partial x}{\partial \eta}=-e^{A} \sin B, \quad \frac{\partial y}{\partial \xi}=e^{A} \sin B, \quad \frac{\partial y}{\partial \eta}=e^{A} \cos B .
$$

Следовательно,

$$
\frac{\partial}{\partial \rho}=\cos \theta \frac{\partial}{\partial \xi}+\sin \theta \frac{\partial}{\partial \eta}, \quad \frac{1}{\rho} \frac{\partial}{\partial \theta}=-\sin \theta \frac{\partial}{\partial \xi}+\cos \theta \frac{\partial}{\partial \eta} .
$$

Вспоминая, что $B+\theta \stackrel{(6)}{=} N$, получаем

$$
\left(\begin{array}{ll}
\frac{\partial x}{\partial \rho} & \frac{\partial x}{\partial \theta} \\
\frac{\partial y}{\partial \rho} & \frac{\partial y}{\partial \theta}
\end{array}\right) \stackrel{(10),(11)}{=} e^{A}\left(\begin{array}{cc}
\cos N & -\rho \sin N \\
\sin N & \rho \cos N
\end{array}\right)
$$

в силу чего (вводя обозначения $\rho_{x}=\partial \rho / \partial x, \rho_{y}=\partial \rho / \partial y, \theta_{x}=\partial \theta / \partial x, \theta_{y}=\partial \theta / \partial y$ ) имеем

$$
\left(\begin{array}{ll}
\rho_{x} & \rho_{y} \\
\theta_{x} & \theta_{y}
\end{array}\right)=\left(\begin{array}{cc}
\cos N & \sin N \\
-\frac{1}{\rho} \sin N & \frac{1}{\rho} \cos N
\end{array}\right) e^{-A} .
$$

Остается учесть формулы $u_{x}=U_{\rho} \rho_{x}+U_{\theta} \theta_{x}$ и $u_{y}=U_{\rho} \rho_{y}+U_{\theta} \theta_{y}$.

Авторы благодарны А. М. Денисову за полезные обсуждения.

\section{СПИСОК ЦИТИРОВАННОЙ ЛИТЕРАТУРЫ}

[1] С. Н. Мергелян, УМН, 11:5 (1956), 3-26. [2] М. М. Лаврентьев, Изв. АН СССР. Сер. матем., 20:6 (1956), 819-842. [3] А.Н. Тихонов, В.Я. Арсенин, Методы решения некорректных задач, Наука, М., 1974. [4] В. И. Арнольд, Геометрические методы в теории обыкновенных дифференциальных уравнений, РХД, Ижевск, 2000. [5] A. S. Demidov, M. Moussaoui, Inverse Problems, 20:1 (2004), 137-154. [6] А. С. Демидов, Докл. РА H, 346:6 (1996), 732-734. [7] A.S. Demidov, V. V. Petrova, V. M. Silantiev, C. R. Acad. Sci. Paris Sér. I Math., 323:4 (1996), 353-358.

\section{А. С. Демидов}

Поступило

Московский государственный университет

им. М. В. Ломоносова

E-mail: asd@math.msu.su

\section{Д. А. Платущихин}

Московский государственный университет им. М. В. Ломоносова 